The notion of centrifugal force, like other examples of the so-called 'force of inertia,' is used simply to enable us to treat a body whose particles are not all moving uniformly in straight lines as in statical equilibrium. Thus, by imagining a force following a certain law of intensity acting outwardly from the earth's axis, in co-existence with the force of gravity, we may regard the earth as a stationary body, subject to these forces. It is the resultant of these forces which we commonly regard as the force of gravity; and, the earth having assumed the form of equilibrium, with a surface everywhere normal to this resultant force, there is no more occasion to consider the centrifugal force as acting independently. But, if we choose to do so, then we must regard the radial force of gravity as acting also; and the centrifugal force acting at any point is then balanced by the force which would, if the earth were not in rotation, reduce it to a spherical form. Thus the centrifugal force can create no tension in an ice-cap, and there is nothing in the nature of the forces acting to interfere with the existence of a continuous ice-cap round the pole, whether symmetrically situated or not. Of course, if a mass of ice were piled up at the pole above the spheroidal surface of equilibrium, lateral pressure would exist, but only in the same way that it would under like circumstances in any other part of the earth; and, wherever this pressure met irsufficient resistance, the ice would 'flow' away in glaciers, just as it does from any elevated region of the earth's surface. WM. WooLsey JoHnson.

\section{Digestion experiments.}

An agricultural experiment-station has to contend against the prejudices of a public which demands speedy work and preposterous generalizations rather than accuracy. When a station, therefore, does do work of a scientific character, it is especially desirous of recognition on the part of science, as such recognition not only brings encouragement to the workers, but also has an influence upon the public to educate toward better expectations and wiser demands. What suggests this remark is an article in Science, April 10, entitled ' Error's in digestion experiments', from which the reader would infer that Professor Armsby's experiments upon digestion were the only ones of that character which have been made in this country. As a matter of record, however, I presume the NewYork agricultural experiment-station, in its Bulletin No. Ixxxv., May 17, 1884, is entitled to the claim of having first published the results of a trial upon the digestibility of a ration in part composed, in the one case, of corn-fodder, and, in the other, of the same material ensilaged. In the forthcoming report of the station for 1884, the figures of these trials, as well as of others, will appear in considerable detail.

Geneva, N.Y., April 13.

E. Lewis Sturtevant.

\section{Volcanic dust from south-western Nebraska.}

There were received at the national museum a few weeks since, from a gentleman in Nebraska, samples of a fine white and very sharp dust, supposed by the sender to be of geyser origin. The deposits from which the samples were taken are stated to be semicircular in outline, from four to ten feet in thickness, and of varying grades of fineness, situated on the banks of small streams that flow into the Republican River. The precise localities given are, Furnas county, two miles south of the Republican River, in sections 9 and 10 , township 3 , north range 21 west; and Harlan county, one mile south of the river, sec- tions 10 and 11 , township 2 , north range 20 west; though the writer states that he has also found similar deposits in Kansas, Colorado, and Wyoming.

An examination of the dust with a microscope shows at once that it is not of Geyser origin, being composed almost wholly of minute fragments of pumiceous glass, with only very rarely a small particle of hornblende. Portions of a coarser deposit, associated with the dust, contain numerous rounded fragments of felspar, a part of which at least is triclinic, as shown by twin striations, and hornblende and magnetite particles. The deposits are therefore, without doubt, volcanic dust and sand, owing their present arrangement to the assorting agency of water and atmospheric currents ; and their mineral composition would indicate that the corresponding lava was an andesite.

The matter is deemed of sufficient importance to mention here, from the fact, that, so far as I am aware, no deposits of dust of this nature have heretofore been reported east of the Rocky-Mountain region.

U.S. nat. museum, April 13. Georae P. Merrill.

\section{Hastings's theory of the corona.}

Your reviewer of the 'Report of the eclipse expedition to Caroline Island' has, by an unfortunate expression, so entirely misrepresented my theory regarding the solar corona, that a correction is necessary. Instead of supposing that the "coronal phenomena may be fully accounted for by applying the wellknown principles of diffraction," as he asserts, I demonstrated that these principles completely fail to account for any part of them. What I did do was to prove that Fresnel's theory is not applicable to the case where both source of light and screen are at an indefinitely great distance from the observer; for then the implied constancy of phase of the wave-surfaces certainly does not exist. This limitation of the theory of diffraction does not seem to have been noted before; and it necessarily implies a distribution of light about the moon during a total eclipse which may be like that of the corona. Assuming that the corona is so formed, I show that all its characteristics (with the exception of the occasional filamentous structure, where the indication of the theory is doubtful) may be explained naturally and easily, even the polarization and absence of the Fraunhofer lines.

I may venture to describe briefly two observations of interest given in detail in the report, since they have not been noted in the review, and have been published only in the report. The first is Professor Holden's observation of the so-called 'shadow-bands' seen just before and just after totality, and which so strikingly suggest a diffraction phenomenon. No one before him, so far as I know, has determined with any useful precision their azimuth, nor had it before been recognized that they move in opposite directions at the two epochs. That their azimuths are those of planes tangent to the sun at the points of second and third contacts, is perhaps not of such immediate interest as the observed reversal of motion, since the latter feature excludes the more favored explanation which makes them shadows.

The other point is the proof that the ' $b$ ' group must be regarded as belonging to the same category as $1474 \mathrm{~K}$, the hydrogen lines $\mathrm{H}$ and $\mathrm{K}$; namely, that of bright corona lines. This renders it extremely probable that all the brilliant as well as high chromospheric lines are also coronal lines. The fact must be regarded as a strong indication in favor of the theory advocated.

New Haven, April 13.
C. S. Hastings. 